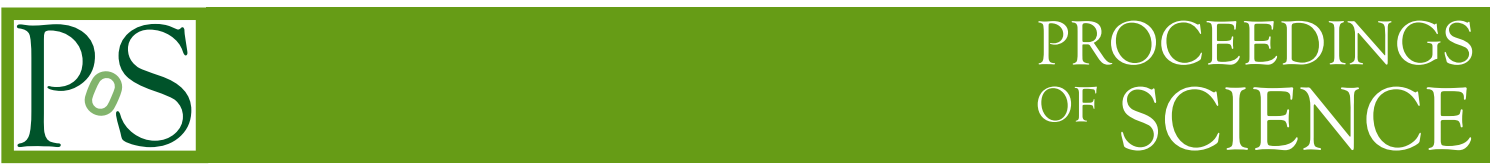

\title{
Testing the MSW Effect in Supernova Explosion with Neutrino Event Rates
}

\author{
Kwang-Chang Lai, ${ }^{a}$ Chun Sing Jason Leung ${ }^{b, *}$ and Guey-Lin $\operatorname{Lin}^{b}$ \\ ${ }^{a}$ Center for General Education, Chang Gung University, \\ Kwei-Shan, Taoyuan, 333, Taiwan \\ ${ }^{b}$ Institute of Physics, National Chiao Tung University, \\ East District, Hsinchu, 300, Taiwan \\ E-mail: jasonleung.py04g@g2.nctu.edu.tw
}

The flavor transition mechanisms of supernova neutrinos as they propagate outward from the deep inside of a supernova are yet to be determined. We study the time-evolution patterns of different neutrino flavors in various flavor transition scenarios. With simulation data of supernova neutrinos, we calculate the neutrino event rates at LArTPC for different flavor transition scenarios. Using the calculated event rates of $v_{e} \mathrm{Ar}$ in liquid argon detector, we calculate the ratio of cumulative time distribution up to $t_{\mathrm{pb}}=100 \mathrm{~ms}$ in several supernova neutrino simulations[1][2][3]. We show that the time evolutions of cumulative ratios are effective in determining whether the MSW oscillations really occur

40th International Conference on High Energy physics - ICHEP2020

July 28 - August 6, 2020

Prague, Czech Republic (virtual meeting)

\footnotetext{
${ }^{*}$ Speaker
} 


\section{Introduction}

Flavor transitions of SN neutrinos have been an attractive field of research and motivated numerous efforts (See [5][6] for a review) on flavor changing during the gravitational core collapse of a massive star. Originating from deep inside the SN core, neutrinos are expected to experience significant flavor transitions as propagating outward to the terrestrial detectors. On account of the MSW effects $v_{e}$ and $\bar{v}_{e}$ fluxes will swap with $v_{x}$ fully or partially when the neutrino vacuum oscillation frequency $\omega=\Delta m^{2} / 2 E$ is of the order of the matter potential, $\lambda=\sqrt{2} G_{F} n_{e}$. Here $\Delta m^{2}$ denotes one of the mass-squared differences, $E$ the neutrino energy, and $n_{e}$ the net electron density. For typical SN post-bounce matter profiles, this the MSW-induced flavor conversions occur at distances of $\sim O\left(10^{3}\right) \mathrm{km}$ from the SN core where $\omega \simeq \lambda$. [5].

Flavor transitions are expected to change flavor compositions of primary SN neutrino fluxes and, hence, to leave imprints in neutrino events measured in terrestrial detectors. This motivates our study of probing flavor transitions with measurements of galactic SN neutrinos at the Earth. Unlike the status of the MSW effects, consensus on collective flavor transitions has not yet been reached so studies of collective flavor transition effects on terrestrial SN neutrino fluxes are few. Meanwhile, the study of fast flavor conversions has just developed in recent years and is still far from a thorough understanding. Therefore, we focus on the MSW effects in SN neutrinos. Based on the understanding that the MSW effects are sensitive to neutrino mass hierarchy, lots of studies are inspired to probe NMH by using SN neutrino events detected at the Earth. These studies all assume the occurrence of the MSW effects. In this work, we, instead, investigate the capability to probe whether the MSW effects occur in SN neutrinos or not. Based on the simulated SN neutrino emissions, we calculate the event rates of $v_{e}$ Ar interaction in liquid argon detector. By comparing the two event rates in the neutronization era, we show that their cumulative ratio is effective in determining the occurrence of the MSW effects for SN neutrinos.

\section{Supernova Neutrino Events and Occurrence of the MSW Effects}

A SN neutrino burst lasts for $\Delta t \approx 10 \mathrm{~s}$, during which the neutronization burst happens at $t_{p b} \sim 10-15 \mathrm{~ms}$. Here, $t_{p b}$ denotes the post-bounce time. In our calculation, we extract the primary neutrino fluxes from the SN simulation [? ] for progenitor masses of 13, 20, and $30 \mathrm{M}_{\odot}$, accounting for $\mathrm{SNe}$ with iron core. With these neutrino fluxes, we calculate event rates of SN neutrinos for all flavors, $v_{e}, \bar{v}_{e}$, and $v_{x}$, whether no oscillations occur to modify the flavor contents or they are changed by the MSW effects as SN neutrinos propagate outward from the core. In the latter case, both normal and inverted hierarchies are taken into consideration. To encmopass the whole duration of the neutronization burst, we perform our analysis for a time period of $\Delta t=0.1 \mathrm{~s}$ from the start. The event rates and quantities induced from these rates are displayed in numbers per bin with a $5 \mathrm{~ms}$ bin width throughout this article. 

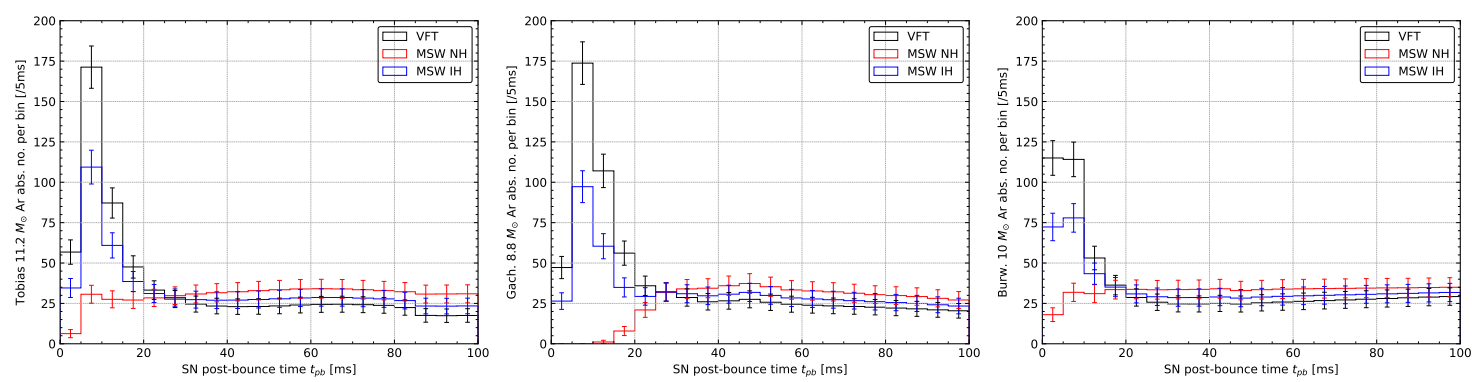

Figure 1: Event rates of $v_{e}$ Ar different flavor transition are shown on the upper and lower panels, respectively. These event rates are obtained in different simulation groups (Fischer's group, Garching group and Burrow's group) with progenitor masses of $11.2,8.8$, and $10 \mathrm{M}_{\odot}$, from left to right.

Assuming a SN at a distance of $5 \mathrm{kpc}$, the event rates of $v_{e} \operatorname{Ar}$ [10] in DUNE [9] is shown in Fig. 1. To account for the sharp rise of $v_{e}$ flux during the neutronization burst, we define cumulative time distributions of the SN neutrino signals for the time interval of interest $t=0-0.1 \mathrm{~s}$ as in [1]

$$
K(t)=\frac{\int_{0}^{t} \frac{d N}{d t} d t}{\int_{0}^{0.1 \mathrm{~s}} \frac{d N}{d t} d t}
$$
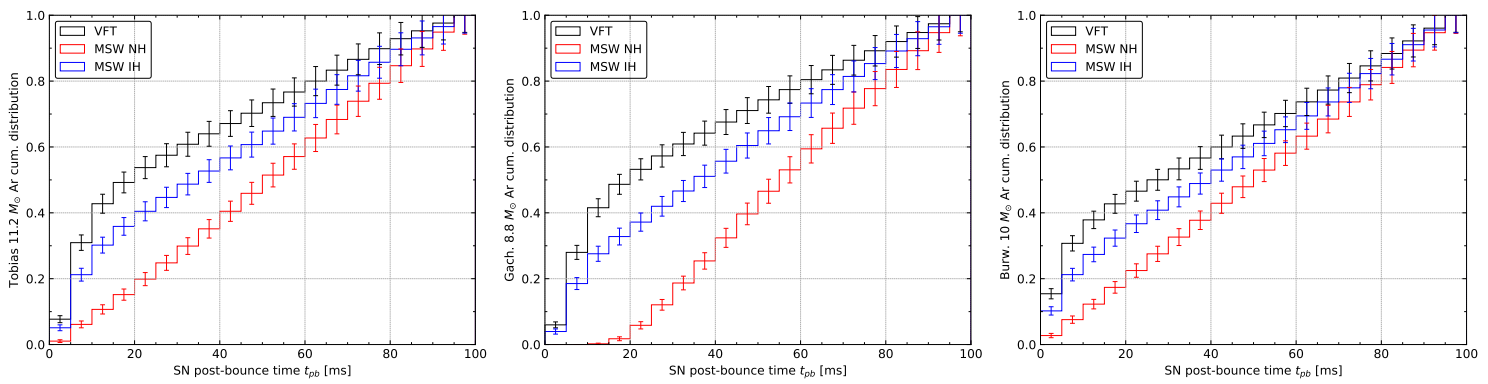

Figure 2: Cumulative distribution of $v_{e}$ Ar signals for the time period of $0.1 \mathrm{~s}$.

In Fig. 2, we show $K_{\mathrm{Ar}}$ the cumulative distributions of $v_{e} \mathrm{Ar}$ in VFT, NH and IH the MSW scenarios. Observe that the cumulative distribution of the MSW IH scenario $K_{\mathrm{Ar}, \mathrm{IH}}$ always in between the others (the MSW NH and VFT). To illustrate that VFT scenario can be distinguished from the MSW scenarios, a ratio between the experimental cumulative distribution, $K_{\mathrm{Ar}}$ and the simulatied cumulative distribution $K_{\mathrm{Ar}, \mathrm{IH}}^{\mathrm{s}}$, is defined as

$$
R_{\mathrm{cum}}\left(K_{\mathrm{Ar}}(t), K_{\mathrm{Ar}, \mathrm{IH}}^{s}(t)\right) \equiv \frac{2}{3}\left(\frac{1+K_{\mathrm{Ar}}(t)}{1+K_{\mathrm{Ar}, \mathrm{IH}}^{s}(t)}\right)-\frac{1}{3}
$$



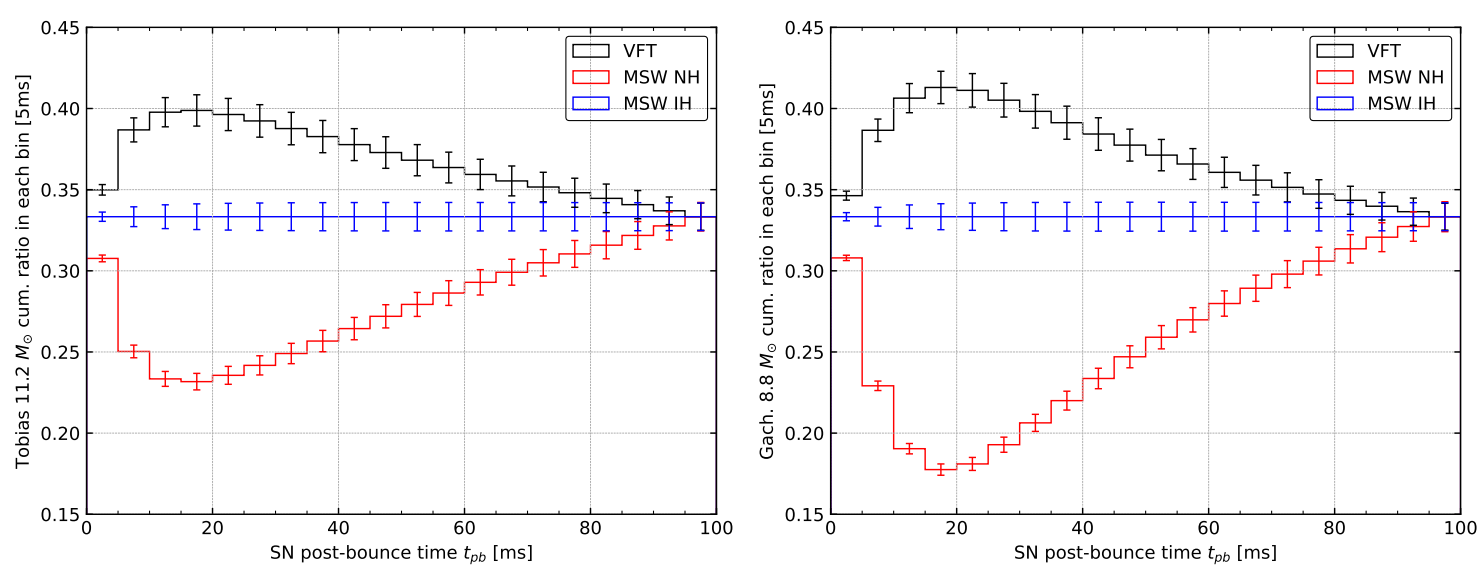

Figure 3: On the left is the cumulative event ratio $R_{\text {cum }}\left(K_{\mathrm{Ar}}^{\mathrm{Gaching}}(t), K_{\mathrm{Ar}, \mathrm{IH}}^{\mathrm{Gachin}}(t)\right)$, and $R_{\text {cum }}\left(K_{\mathrm{Ar}}^{\text {Tobias }}(t), K_{\mathrm{Ar}, \mathrm{IH}}^{\mathrm{Tobias}}(t)\right)$ on the right.

The same simulation data has been used in both the numerator (as an experimental data) and denominator (as a simulation) of the cumulative event ratio eq.(2) for demonstrative convenience (say $\left.R_{\text {cum }}\left(K_{\mathrm{Ar}}^{s}(t), K_{\mathrm{Ar}, \mathrm{IH}}^{s}(t)\right)\right)$ in Fig.3. It is clear that a non-zero function divided by itself will result in a constant function, the treatment in eq.(2) is an continues deformation such that the MSW IH curve in $R_{\text {cum }}$ becomes a horizontal straight line as shown in Fig. 3. Obviously, preliminary discriminating criteria can be made by observing the time evolution patterns of these curves.

- $\mathrm{VO}$ : concave curve

- MSW IH : a horizontal straight line with a value $1 / 3$

- MSW NH : convex curve

The horizontal straight line with a value $1 / 3$ in the MSW IH scenario is due to the fact that we have taken the same value in both the $R_{\text {cum }}$ numerator (experimental data) and denominator (simulation data) in the MSW IH scenario as explained above. However, practically there must be some differences between the experimental and simulation data, this causes the $R_{\mathrm{cum}}\left(K_{\mathrm{Ar}, \mathrm{IH}}(t), K_{\mathrm{Ar}, \mathrm{IH}}^{s}(t)\right)$ to deviate from the horizontal straight line, therefore, the above criteria have to be slightly modified. In Fig.4, Garching (left) and Tobias (right) simulations have been taken as experimental data and placed in the numerator of the cumulative event ratio, on the other hand, Burrows' SN neutrino simulation data is placed in the denominator (say $R_{\text {cum }}\left(K_{\mathrm{Ar}, \mathrm{IH}}^{\mathrm{Garchin} / \mathrm{Tobias}}(t), K_{\mathrm{Ar}, \mathrm{IH}}^{\mathrm{Burrows}}(t)\right)$ ). Although the $R_{\text {cum,IH }}(t)$ no longer manifests as the previous horizontal straight line, modified discriminating criteria can be constructed from the time evolution of these new curves.

- $\mathrm{VO}$ : concave curve with a local maximum greater than 0.4

- MSW IH : concave curve lies in between 0.3 and 0.4

- MSW NH : convex curve 

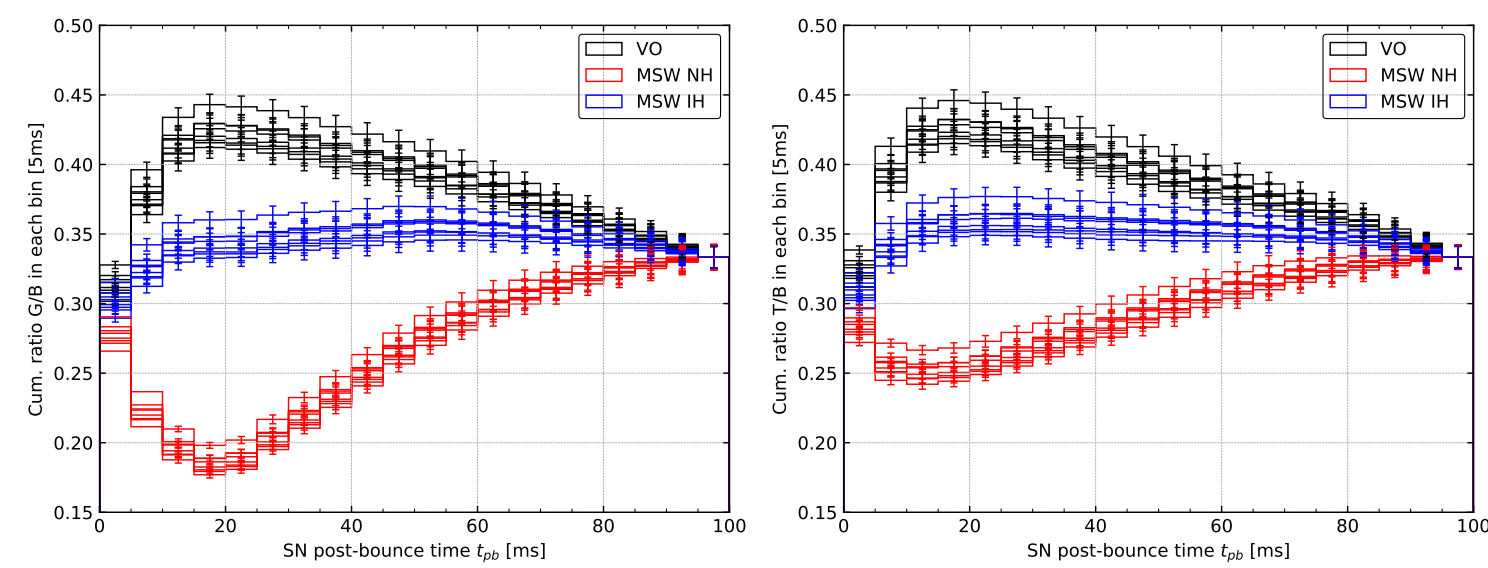

Figure 4: On the left is the cumulative event ratio $R_{\text {cum }}\left(K_{\mathrm{Ar}}^{\mathrm{Garching}}(t), K_{\mathrm{Ar}, \mathrm{IH}}^{\mathrm{Burows}}(t)\right)$, and $R_{\text {cum }}\left(K_{\mathrm{Ar}}^{\text {Tobias }}(t), K_{\mathrm{Ar}, \mathrm{IH}}^{\mathrm{Burrows}}(t)\right)$ on the right. Lines with the same colour indicate different SN progenitor masses in Burrows' simulation.

\section{Summary and Conclusions}

We have proposed a method to testify that the occurrence of the MSW effects in SN neutrinos by measuring the of SN neutrino event rates during the neutronization burst in liquid argon detector. $v_{e} A r$ events in liquid argon detector is taken to define a cumulative event ratio $K_{r}$. The ratios are calculated with SN neutrino emissions extracted from SN simulation data. With this ratio, we have shown whether the MSW effects occur for SN neutrinos or not can be probed by comparing the time evolutions of $v_{e}$ Ar event rates in liquid argon detector.

\section{References}

[1] Tobias Fischer, Lorenz Hudepohl et al., "Probing the neutrino mass hierarchy with the rise time of supernova burst", Phys.Rev. D 85085031

[2] P. D. Serpico, S. Chakraborty, T. Fischer, L. Hudepohl, H. T. Janka and A. Mirizzi, Phys.Rev. D 85 (2012) 085031, arXiv:1111.4483

[3] A. Burrows, D. Radice and D. Vartanyan, Mon. Not. Roy. Astron. Soc. 485 (2019) no.3, 3153,arXiv:1902.00547

[4] P.A. Zyla et al. (Particle Data Group), to be published in Prog. Theor. Exp. Phys. 2020, 083C01 (2020)

[5] L. Wolfenstein, Phys. Rev. D 17, 2369 (1978).

[6] A. S. Dighe and A. Y. Smirnov, Phys. Rev. D 62, 033007 (2000), arXiv:hep-ph/9907423.

[7] A. Strumia and F. Vissani, Phys. Lett. B 564 (2003) 42-54, astro-ph/0302055.

[8] F. An et al. [JUNO Collaboration], J. Phys. G 43, no. 3, 030401 (2016), arXiv:1507.05613. 
[9] DUNE Conceptual Design Report, arXiv:1512.06148v2 [physics.ins-det]

[10] I. Gil-Botella, A. Rubbia, "Oscillation effects on supernova neutrino rates and spectra and detection of the shock breakout in a liquid Argon TPC", JCAP0310:009,2003

[11] Kwang-Chang Lai, C. S. Jason Leung and Guey-Lin Lin, arXiv:2001.08543 [astro-ph.HE] 